\title{
A molecular-level computational study of the diffusion and solubility of water and oxygen in carbonaceous polyethylene nanocomposites
}

Edvin Erdtman, Martin Bohlén, Peter Ahlström, Thomas Gkourmpis, Mikael Berlin,

Thorbjörn Andersson and Kim Bolton

\section{Journal Article}

\section{Tweet}

N.B.: When citing this work, cite the original article.

Original Publication:

Edvin Erdtman, Martin Bohlén, Peter Ahlström, Thomas Gkourmpis, Mikael Berlin, Thorbjörn Andersson and Kim Bolton, A molecular-level computational study of the diffusion and solubility of water and oxygen in carbonaceous polyethylene nanocomposites, Journal of Polymer Science Part B, 2016. 54(5), pp.589-602.

http://dx.doi.org/10.1002/polb.23951

Copyright: Wiley: 12 months

http://eu.wiley.com/WileyCDA/

Postprint available at: Linköping University Electronic Press

http://urn.kb.se/resolve?urn=urn:nbn:se:liu:diva-129607

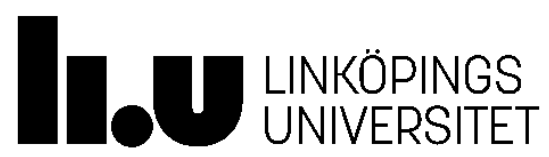




\title{
A molecular-level computational study of the diffusion and solubility of water and oxygen in carbonaceous polyethylene nanocomposites
}

\author{
Edvin Erdtman, ${ }^{1}$ Martin Bohlén, ${ }^{1}$ Peter Ahlström, ${ }^{1}$ Thomas Gkourmpis, ${ }^{2}$ Mikael Berlin, ${ }^{3}$ Thorbjörn \\ Andersson, ${ }^{3}$ Kim Bolton ${ }^{1}$ \\ ${ }^{1}$ School of Textiles, University of Borås, Allégatan 1, 50190 Borås, Sweden \\ ${ }^{2}$ Innovation \& Technology, Borealis AB, 44486 Stenungsund, Sweden \\ ${ }^{3}$ Tetra Pak Packaging Solutions AB, Ruben Rausings gata, 22186 Lund, Sweden \\ Correspondence to: Martin Bohlén (E-mail: martin.bohlen@hb.se)
}

\begin{abstract}
Monte Carlo and molecular dynamics simulations were performed to investigate the effect on the solubility, diffusion and permeability of water and oxygen when adding graphene or single-walled carbon nanotubes (SWCNTs) to polyethylene (PE). Compared to pure PE, addition of graphene lowered the solubility of water while, at lower temperatures, the oxygen solubility increased due to the oxygen - graphene interaction. Addition of SWCNTs lowered the solubility of both water and oxygen compared to pure PE. A detailed analysis showed that an ordered structure of PE is induced near the additive surface which leads to a decrease in the diffusion coefficient of both penetrants in this region. The addition of graphene does not change the permeation coefficient of oxygen (in the direction parallel to the filler) and, in fact, may even increase this coefficient compared to pure PE. In contrast, the water permeability is decreased when graphene is added to PE. The addition of SWCNTs decreases the permeability of both penetrants. Graphene can consequently be added to selectively increase the solubility and permeation of oxygen over water, at least at lower temperatures.
\end{abstract}

KEYWORDS: Solubility; Diffusion; Polyethylene; Nanocomposites; Molecular modelling.

\section{INTRODUCTION}

The ability to control the permeation of water and oxygen through polymer materials has many benefits and can improve the efficiency of products used in applications such as fuel cells, ${ }^{1}$ gas separation ${ }^{2}$ and various packaging materials. ${ }^{3}$ Controlling the permeation of these two penetrants is of utmost importance for the food industry since they are critical for the shelf-life of the packaged products. Water controls the consistency and structure of many foodstuffs. Also, the rate of many (degrading) chemical reactions is increased when they occur in aqueous solution. Oxygen is, for example, the cause of food spoilage through oxygenation. Furthermore, permeation of both water and oxygen must be prevented to inhibit the growth of moulds and yeasts on the packaged product.

Polyethylene (PE) is one of the most thoroughly studied polymers and is frequently used in the packaging industry. Both high density (HDPE) and low density polyethylene (LDPE) are easily 
produced and processed at an industrial scale, with density playing a critical role on the resin's properties. ${ }^{4}$ HDPE has been seen to exhibit better barrier properties for water and oxygen compared to LDPE. ${ }^{4,5}$ Nonetheless, oxygen permeates through HDPE $^{4,5}$ which, as discussed above, can have a negative impact on packaged products. In terms of moisture barrier both LDPE and HDPE are considered to be reasonably good. ${ }^{4-6}$

The permeation coefficient is the product of the solubility and diffusion coefficients. Both of these coefficients depend on the temperature and have been studied using experimental ${ }^{7}$ and theoretical methods. ${ }^{8,9}$ For example, Michaels et al. ${ }^{10,11}$ reported that the solubility of oxygen in PE increases slightly between 285 and $315 \mathrm{~K}$, and that a further increase in temperature results in a larger increase in solubility. Other studies have shown that the solubility of oxygen decreases when increasing the temperatures up to $315 \mathrm{~K}$, and that a further increase in temperature has little effect on the solubility. ${ }^{12}$ McCall et al. ${ }^{13}$ reported that the solubility of water in PE increases between 298 and $335 \mathrm{~K}$. Diffusion of water and oxygen has also been studied and the results show that there is an increase in diffusion rate of both penetrants with increasing temperature (up to $335 \mathrm{~K}$ ). ${ }^{12-15}$

Börjesson et al. ${ }^{6}$ performed a systematic study of the solubility, diffusion and permeation of water and oxygen in PE between 278 and $308 \mathrm{~K}$ (relevant for the storage of packaged foodstuffs) using Monte Carlo (MC) and molecular dynamics (MD) simulation techniques. They found that the solubility coefficient of oxygen in PE is six orders of magnitude larger than that of water $((0.3 \pm$ $0.03) \mathrm{cm}^{3} \mathrm{~cm}^{-3}$ at $278 \mathrm{~K}$ for oxygen compared to $(0.26 \pm 0.16) \times 10^{-6} \mathrm{~cm}^{3} \mathrm{~cm}^{-3}$ for water) and that the diffusion coefficient is similar for both penetrants $\left((0.6 \pm 0.3) \times 10^{-6} \mathrm{~cm}^{2} \mathrm{~s}^{-1}\right.$ for water and $(0.69 \pm 0.27) \times 10^{-6} \mathrm{~cm}^{2} \mathrm{~s}^{-1}$ for oxygen). These authors also report that the solubility coefficient of oxygen decreased slightly with increasing temperature, which is in agreement with some previous studies ${ }^{15,16}$ while contradicting results from other studies where the opposite trend was found. ${ }^{12,14}$ The solubility coefficient of water increased with increasing temperature, which is in agreement with several studies. ${ }^{13,17}$ Since the permeation coefficient is calculated as the product of the solubility and diffusion coefficients, the permeation coefficient of oxygen was also six orders of magnitude larger than for water. ${ }^{6}$

One way to control the permeability of water and oxygen through PE is to include additives in the matrix. ${ }^{18}$ These additives can act either as physical barriers, ${ }^{19}$ affect the structure $^{8,9,20,21,22,23}$ and dynamics ${ }^{23,24,25}$ of the polymer matrix or bond physically or chemically with the penetrant molecules.

Nano-sized high aspect ratio carbon allotropes like graphene or carbon nanotubes (CNTs) are reported to have unique properties and their inclusion in polymer matrices has the potential to lead to the creation of tailor-made systems with superior mechanical, electrical and barrier properties. $^{26,27}$

Introducing relatively rigid additives like these in polymer systems induces confinement effects in the surrounding polymer chains. ${ }^{22-25}$ Both the structural (static) and the dynamic behavior of confined polymer matrices have been studied extensively. ${ }^{8,9,20-25}$ The effect on the polymer structure is dependent on the interactions between the polymer and the additive. An attractive interaction leads to an increase in monomer density in the vicinity of the additive, compared to the bulk, while a repulsive interaction results in a depletion in the vicinity of the additive. ${ }^{22}$ Slower dynamics (and thus an increased glass transition temperature, $T_{\mathrm{g}}$ ) close to the confining surface has been reported when there are attractive interfacial forces between the additive surface and the polymer chains. ${ }^{22,24,25}$ The dynamics is hence slower in this region than in the bulk and a gradient between the two regions can exist. ${ }^{24}$ Also, when the distance between two confining surfaces (or additives) is decreased the gradient 
disappears due to the overlap of the immobilized regions but, interestingly, the overall mobility increases.${ }^{24}$ Weak or repulsive interactions have been reported to increase the dynamics and hence lower the $\mathrm{Tg}_{\mathrm{g}}{ }^{25}$ As discussed below, the static and dynamic properties of the polymer matrix affects the solubility, diffusion and permeation of water and oxygen in the studied nanocomposites.

Furthermore, graphene and CNTs are impermeable to water and oxygen and can therefore act as physical barriers, creating a tortuous path for the diffusing molecules that would lead to a decrease in diffusion rate. ${ }^{19}$ However, the penetrant molecules may also have a stronger binding to the additives than the polymer, which could result in a higher solubility. This is especially true if the graphene sheets or CNTs have been functionalized with chemical groups that can interact strongly with the penetrant molecules. Furthermore, the inclusion of graphene or CNTs can also alter the structure of the polymer matrix. The polymer filler interaction has been seen to create morphological changes that can lead to significant ordering of the macromolecular chains along the various interfaces. ${ }^{27,28}$ These structural changes are present for simple fillers like carbon black, ${ }^{29}$ but have the potential to become more pronounced for high aspect ratio fillers, affecting a number of properties including solubility and/or diffusion. ${ }^{8,9}$ For example, a quasi-crystalline structure of the $\mathrm{PE}$ was predicted to form around the CNT in a simulation study by Herasati et al. ${ }^{20}$ In another simulation study it was estimated that the diffusion of PE chains increased in the vicinity of a CNT in comparison to the bulk. ${ }^{21}$

Several experimental studies have investigated the effect of including nanostructured additives on solubility, diffusion and permeation in polymer materials.,30-32 For example, the permeability of gases in a PE-CNT nanocomposite was found to be lower than in pure $\mathrm{PE}^{30}$ For oxygen the permeation coefficient decreased from 12 to $6 \mathrm{~mL} \mathrm{~cm}$ day $^{-1}$ $\mathrm{m}^{-2}$ atm $^{-1}$ when 2.5 wt\% CNT was added. Similarly, the permeability of water and oxygen in a composite of polypropylene (PP) with $1 \mathrm{wt} \%$ CNT decreased by $8-9 \%$ compared to pure $\mathrm{PP}^{31}$ On the contrary to $\mathrm{PE}$ and PP, oxygen diffusion in a composite of multi-walled CNTs (MWCNTs) and polystyrene (PS) was found to be higher than in pure PS. ${ }^{32}$ Addition of graphite nanoplatelets to polyethylene terephthalate increased the crystallinity and decreased the oxygen permeation. ${ }^{7}$

Hence, addition of carbonaceous nanostructures to polymers affects the permeation of water and oxygen. To gain further insights and to obtain a molecular level understanding of the solubility, diffusion and permeation mechanisms, experimental work can be complemented with simulation studies. An increased understanding of these mechanisms can assist in identifying new materials with improved, and even tailor-made, permeation properties.

Simulation studies can be performed using a variety of methods. First principle methods are fairly accurate, but computationally expensive and are therefore used mostly for very small systems and / or short simulations. MC and MD methods based on classical mechanics are used to model far larger systems. In this study first principles methods are used to validate the choice of force field used for the MC and MD simulations. An advantage of simulation studies compared to experimental work is that the system being investigated can be precisely defined in terms of its composition (and can hence be free from impurities). The atomic motion is also readily monitored and analysed. Therefore, the importance of a specific process (e.g. addition of graphene/CNTs on water/oxygen diffusion) can be studied in a detailed and precise way at the molecular level. This molecular-level precision and analysis is often missing from experimental studies, and when comparing results from simulation and experimental studies it is important to account for the fact that the model system used in 
simulations is different from the experimental system, where impurities, voids and crystalline regions are usually present.

To the best of our knowledge, this work consists of the first systematic study of how graphene and SWCNTs affect the PE structure and the permeation of small penetrants, such as water and oxygen, through the nanocomposite. Since the additives used are impermeable to the penetrants, the aim of the study is to obtain a molecular-level understanding of how they influence the solubility and the diffusion parallel and perpendicular to their axes. The results for the composites are compared to an earlier study of pure $\mathrm{PE}^{6}{ }^{6}$ that uses the same computational methods as those used here. The permeability of water and oxygen was studied by separate simulations of the solubility and diffusion in the composites. The temperature range was 278 to $328 \mathrm{~K}$, which covers the range from refrigerator to slightly above room temperature that is usually used for storage of packaged foods.

The impermeable nature of graphene and SWCNTs decreases the permeation in the direction perpendicular to the graphene sheet and/or SWCNT central axis. The permeation in this direction will be the focus of a future study where non-periodic graphene sheets and SWCNTs will be used as additives. However, it should be iterated that the permeation of oxygen and water in the direction parallel to the graphene sheet and SWCNT central axis also is of importance and influences the results as discussed below.

\section{METHOD}

The AMBER (Assisted Model Building and Energy Refinement) force field ${ }^{33}$ is used to describe the systems. This force field was validated for pure PE systems by comparing the densities of $n$-octane and PE obtained from AMBER with experimental values of $n$-octane and amorphous PE. ${ }^{6}$ For the composite systems described below, AMBER is validated by comparing the results with those of first principle methods using a small model system.

\section{Force field validation}

Since both graphene and SWCNTs contain $\mathrm{sp}^{2}$ carbon atoms, it is expected that the AMBER force field will be valid for the graphene systems if it is valid for the SWCNT systems. Testing the force field was therefore limited to systems containing SWCNTs. Systems consisting of a $(3,3)$ SWCNT with hydrogen terminated ends (60 atoms in total) and a hexane molecule were used. To find a chemically relevant minimum on the potential energy surface, three models with very different alignments of the SWCNT and the hexane molecule were constructed. As shown in Figure 1, in the first configuration the hexane molecule is parallel to the SWCNT wall, in the second configuration it is perpendicular to the SWCNT wall and in the third configuration it is at the end of the SWCNT. These configurations were geometry optimised and annealed using the AMBER force field as implemented in the Large-scale Atomic/Molecular Massively Parallel Simulator (LAMMPS) $^{34}$ program package. For each configuration, the annealing simulations were performed over 30 cycles with initial and final temperatures of $1 \mathrm{~K}$ and a mid-cycle temperature of $7 \mathrm{~K}$. Each cycle consisted of $10^{4}$ MD steps of $1.0 \mathrm{fs}$ each and after each cycle was the structure geometry optimised using the Hessian-free truncated Newton, a steepest descent and the conjugate gradient algorithms with energy convergence criteria of $2.09 \times 10^{-4} \mathrm{~kJ}$ $\mathrm{mol}^{-1}$ and force convergence criteria of $4.18 \times 10$ $6 \mathrm{~kJ} \mathrm{~mol}^{-1} \AA^{-1}$. The lowest energy structure obtained from these simulations was a structure where the hexane molecule was located along the SWCNT wall as shown in the lower panel of Figure 1. It should be noted that this configuration is very similar to the structure obtained from a previous study ${ }^{35}$ where the same model system was used to validate the COMPASS force field ${ }^{36}$ and where a similar annealing procedure was used to localize the global minimum of the potential energy 
surface. ${ }^{35}$ The structure with the lowest energy was further optimised using dispersion corrected density functional theory (DFT-D) with the B3LYP ${ }^{37}$ hybrid functional and the 6$311 G(d, p)$ and $6-311 G(2 d, 2 p)$ basis sets. The dispersion corrections can be of importance for systems like the ones studied here, so the correction of Grimme et al. ${ }^{38,39}$ has been used. This method has been found to account for dispersion effects for a large variety of systems ${ }^{40}$ and has previously been used for systems similar to the PE-SWCNT systems studied here. ${ }^{41,42}$ All DFT-D geometry optimisations were performed using the GAMESS-US (General Atomic and Molecular Electronic Structure System $)^{43,44}$ using the quadratic approximation method with a gradient convergence tolerance of $4.98 \times 10^{-2} \mathrm{~kJ}$ $\mathrm{mol}^{-1} \AA^{-1}$ and a RMS gradient tolerance of $1.65 \times$ $10^{-2} \mathrm{~kJ} \mathrm{~mol}^{-1} \AA^{-1}$.

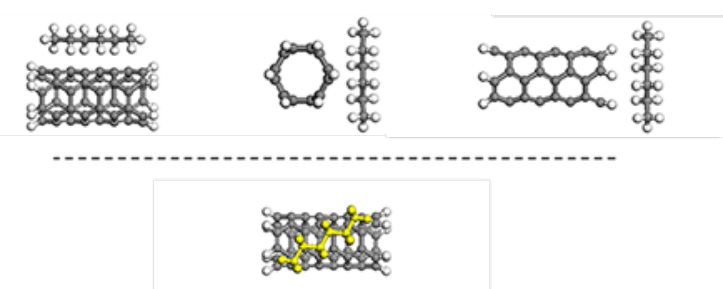

FIGURE 1. Initial PE-SWCNT structures used to test the validity of the AMBER force field (upper panel) and the lowest energy structure obtained after annealing and geometry optimisation with the AMBER force field (lower panel).

\section{Preparation of PE nanocomposite systems}

The PE-graphene systems were prepared as follows: An infinitely large graphene sheet was built in the $x$-y plane of a periodic box where each periodic image consisted of 416 carbon atoms. The size of the periodic box was $34.08 \times$ $31.97 \times 30.00 \AA$ in the $x-, y$ - and $z$-dimensions, respectively. This box size is sufficiently large to prevent interaction between an atom and its periodic image. Six PE chains, each consisting of a backbone of 200 atoms, were randomly inserted with the grand-canonical MC (GCMC) method using the Monte Carlo for Complex Chemical Systems (MCCCS) Towhee ${ }^{45}$ software. This length and number of PE chains has been used in previous studies and has been reported to be sufficient to provide the correct trends of the properties studied here. ${ }^{46}$

The PE-SWCNT systems were prepared in a similar manner. Synthesised SWCNTs typically have diameters of $\sim 10 \AA$ to $30 \AA{ }^{47}$ and an armchair $(12,0)$ SWCNT with a diameter of 9.2 $\AA$ as chosen for the simulations (a thin SWCNT that limits the computational expense while still being relevant to experimental investigations). The chirality of the SWCNT is not expected to affect the trends in solubility, diffusion and permeation reported here, since the strength of the interactions between the SWCNT and the $\mathrm{PE}$ or penetrant is not expected to be sensitive to the SWCNT chiral numbers.

A $(12,0)$ SWCNT was accordingly built along the z-direction of a periodic box. Each periodic image consisted of 384 carbon atoms and the $x$ , $y$ - and z-dimensions of the periodic box were $30.00 \times 30.00 \times 33.60 \AA$. Six PE chains were inserted in the same way as for the graphene systems described above.

The periodic boxes described above result in ratios of $22.9 \mathrm{wt} \%$ for the graphene systems and $21.5 \mathrm{wt} \%$ for the SWCNT systems. These are high compared to many experimental studies where additive amounts are usually less than 5 wt\%. ${ }^{48,49}$ These ratios were chosen to enable computational studies in a tractable time and to identify any possible effects of the additives on PE structure and penetrant solubility and diffusion. Hence, if either the graphene or SWCNT additives show an effect in these simulations, the magnitude of the effect is likely to be smaller in the experimental systems (although the effects and the molecular-level mechanisms of the effects will be the same as those revealed here).

Thirty six different systems for each nanocomposite were prepared using the GCMC 
method discussed above. Each system was equilibrated using MD simulations in the NVT and NpT ensemble using the LAMMPS software, following the same procedure used in an earlier study of pure amorphous PE. ${ }^{6}$ The systems were considered to be equilibrated when the timeaveraged density and energy were constant. The equilibrated densities of the graphene- and SWCNT-containing systems at $298 \mathrm{~K}$ were 0.99 $\mathrm{kg} \mathrm{dm}^{-3}$ and $0.96 \mathrm{~kg} \mathrm{dm}^{-3}$, respectively. As discussed below, this is in good agreement with previously reported theoretical predictions and experimental data. ${ }^{35,50}$ Examples of equilibrated systems are shown in Figure 2. When equilibrated, each of these 36 systems was simulated for a further 1 ns. Four snapshot structures were taken at 250 ps intervals from each simulation to get $144 \mathrm{PE}$-graphene and 144 PE-SWCNT structures, which were used as input structures for the solubility and diffusion simulations. As shown by the error bars below (95\% confidence intervals), this is sufficient to obtain statistically converged results.

\section{Solubility}

The solubility coefficient (S) was calculated by performing $M C$ simulations in the Gibbs NpT ensemble (GEMC) using the MCCCS Towhee software and the AMBER force field at 101.325 $\mathrm{kPa}$ and at temperatures of 278, 288, 298, 308, 318 and $328 \mathrm{~K}$. The liquid water and gaseous oxygen containing reservoir boxes contained 1000 and 200 water or oxygen molecules, respectively.

Five dummy atoms were inserted into the SWCNT to prevent penetrant molecules from entering this region (SWCNTs are usually capped under experimental conditions, which prevents penetration of molecules). A LennardJones (L-J) potential was applied to the dummy atoms with the same well depth parameter, $\varepsilon$, as oxygen in the AMBER force field. The interatomic distance parameter, $\sigma_{\text {dummy, }}$ was chosen to fill the inner space of the SWCNT. The SWCNT diameter after equilibration was $9.2 \AA$. The $\sigma$ of the carbon atoms in the SWCNT is 3.4
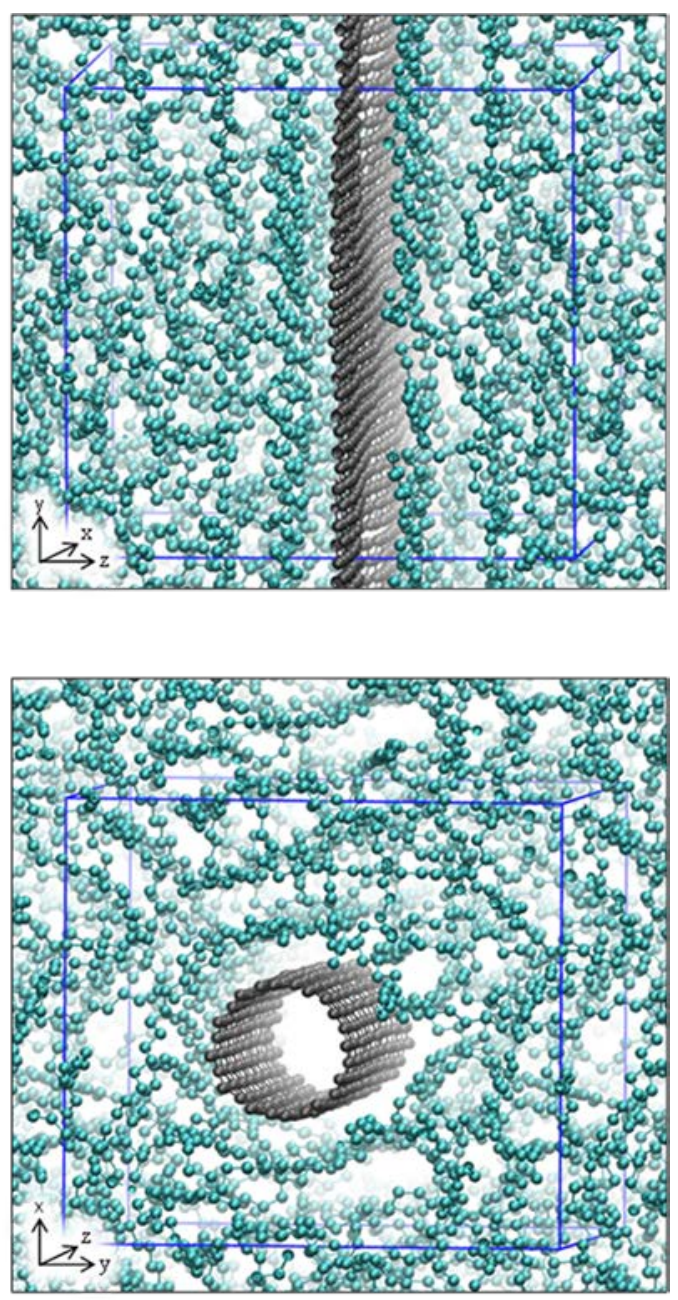

FIGURE 2. Equilibrated structures of PEgraphene (a) and PE-SWCNT (b) nanocomposites. The PE hydrogen atoms are omitted for the sake of clarity.

$\AA$, and hence $\sigma_{\text {dummy }}$ was set to $9.2-3.4 \AA=5.8$ $\AA$.

The GEMC simulations had $2 \times 10^{7}$ and $2 \times 10^{8}$ steps when calculating the water and oxygen solubility coefficients, respectively. A smaller number of steps was used for the water to allow for results in a computationally tractable time (the water box contains five times more molecules compared to the oxygen box and there are electrostatic interactions between the water molecules). Nevertheless, these simulations allowed for equilibration (seen by a constant penetrant density in the polymer box) and production of data. The probability of 
performing a volume change move on the penetrant box was 0.01 . The probability of performing a penetrant transfer move between boxes was 0.01 . The probability of performing an atom translation move on any of the molecules was 0.48 and the probability for each molecule type was $0.1,0.8$ and 0.1 for the graphene, PE and penetrant, respectively. Centre-of-mass translations and rotations along the centre of mass for the water or oxygen molecules were selected with probabilities of 0.25 each.

The unit for $S$ is the volume fraction $\left(\mathrm{cm}^{3}\right.$ (penetrant) $\mathrm{cm}^{-3}$ (polymer)). The penetrant volume $\left(\mathrm{cm}^{3}\right.$ penetrant) is obtained by calculating the number of penetrant molecules in the polymer box and then determining the volume that these molecules would have had in the reservoir box (the effective volume). The polymer volume $\left(\mathrm{cm}^{3}\right.$ polymer) is the volume of the polymer box. Hence, the solubility coefficient is the partition function for the penetrant molecules.

The unit of $\mathrm{S}$ used in this work $\left(\mathrm{cm}^{3}\right.$ (penetrant) $\mathrm{cm}^{-3}$ (polymer) is related to the unit of $\mathrm{cm}^{3}$ (STP) $\mathrm{cm}^{-3}$ (atm) which is also used in the literature and describes the volume of oxygen or water at STP conditions adsorbed in a certain volume of polymer. The conversion between the two units for water and oxygen is:

$$
\begin{aligned}
& S\left[\frac{\mathrm{cm}^{3}(\mathrm{STP})}{\mathrm{cm}^{3} \mathrm{~atm}}\right]_{\text {oxygen }}=S\left[\frac{\mathrm{cm}^{3}}{\mathrm{~cm}^{3}}\right] \frac{T_{0}}{T p_{0}} \\
& S\left[\frac{\mathrm{cm}^{3}(\mathrm{STP})}{\mathrm{cm}^{3} \mathrm{~atm}}\right]_{\text {water }}=S\left[\frac{\mathrm{cm}_{\text {liquid water }}^{3}}{\mathrm{~cm}^{3}}\right] \frac{\rho_{\text {water }}}{p_{\text {vap }} \rho_{\text {vap }, 0}}
\end{aligned}
$$

where $T$ denotes the temperature, $p_{\text {vap }}$ the (partial) pressure of water vapour and $\rho$ the density. A subscripted zero denotes STP conditions ( $T=273.15 \mathrm{~K}$ and $\mathrm{p}=101.325 \mathrm{kPa})$.

\section{Diffusion}

The diffusion coefficient (D) was obtained by performing MD simulations with one penetrant molecule (water or oxygen) in each of the 144 composite systems using the LAMMPS software. The penetrant molecule was randomly inserted into the composite using the GCMC method described above.

For each structure the MD simulations were performed for $4.0 \mathrm{~ns}$ in the NVT ensemble using the Nosé-Hoover thermostat ${ }^{51,52}$ with a damping coefficient of $0.1 \mathrm{ps}$, and a time step of $1.0 \mathrm{fs}$. The Verlet integration algorithm, which has the strength of being time-reversible ${ }^{53}$ was used.

D was calculated from the slope of the mean square displacement (MSD) over time for the diffusing molecule as in equation 1 :

$$
D=\frac{d(M S D(t))}{2 n d t}
$$

where $\mathrm{n}$ is the number of dimensions of the MSD. The MSD was calculated as both a time and an ensemble average, with the MSD analyzer MATLAB code, ${ }^{54}$ as follows:

$M S D(t)=\frac{\sum_{i}^{N} M S D_{i}(t)}{N}$

where $N$ is the number of trajectories (144) and

$\operatorname{MSD}_{i}(t)=\left\langle\left|r_{i}(t)-r_{i}(0)\right|^{2}\right\rangle$

where $\boldsymbol{r}_{i}(t)$ is the position of the molecule at time $t$ and $<>$ denotes the ensemble average.

The slope was calculated from the first $30 \%$ of the trajectory, but excluding the first 20 ps (i.e., it was calculated from $t=20$ to 1200 ps of the 4.0 ns trajectory). Due to the fact that the penetrant molecules initially move freely (in a void) which gives a $D$ that is too large, the first 20 ps of the simulation were excluded. The final $70 \%$ was removed due to statistical uncertainty in the data. 


\section{Local Diffusion}

The local diffusion (at set distances from the additive) was calculated as described above, but by dividing the trajectories into shorter time intervals (500 ps) and calculating the diffusion in each interval. The length of the intervals was chosen so that very few molecules had time to "jump" between the layers of PE around the graphene or SWCNT, but long enough to get reliable statistics of the diffusion parallel to the additive. The average distance from the additive surface to the penetrant was calculated for each time interval, and classified into one of three regions associated with the density distribution of the penetrant (the location of those regions will be shown in the Results section below). The average $D$ was calculated for each interval.

This method gives higher values of $D$ compared to the calculations utilizing the 1180 ps long interval of the trajectories described above, due to the faster movement of the penetrant molecules in the beginning of each MSD analysis.

\section{Permeability}

The permeation coefficient $(P)$ is calculated according to equation 4 :

$P=D S$

where $D$ is in units of $\mathrm{cm}^{2} \mathrm{~s}^{-1}$ and $S$ in $\mathrm{cm}^{3} \mathrm{~cm}^{-3}$. Hence, $P$ has units of $\mathrm{cm}^{2} \mathrm{~s}^{-1}$. If $D$ is the diffusion parallel to the filler, $P$ will be a measurement of the permeation of the water or oxygen molecule through the composite parallel to the filler.

\section{Temperature dependence}

The van't Hoff equation can relate the solubility coefficient $(\mathrm{S})$ to the enthalpy $\left(\Delta \mathrm{H}_{\mathrm{S}}\right)$ and entropy $\left(\Delta \mathrm{S}_{\mathrm{S}}\right)$ of solution according to:

$$
\ln S=\frac{\Delta S_{S}}{R}-\frac{\Delta H_{S}}{R} \frac{1}{T}
$$

where $R$ is the gas constant and $T$ is the temperature.

From $\Delta \mathrm{H}_{\mathrm{S}}$ and $\Delta \mathrm{S}_{\mathrm{s}}$ the Gibbs' free energy of solution $\left(\Delta \mathrm{G}_{\mathrm{S}}\right)$ can be calculated according to:

$\Delta G_{S}=\Delta H_{S}-T \Delta S_{S}$

In analogy to the van't Hoff equation, the Arrhenius equation can be used to calculate the activation energy for diffusion and permeation. Although this was done, the results are not presented here since the statistical uncertainties are too large to allow for meaningful conclusions.

\section{RESULTS}

\section{Force field validation}

The DFT-D binding energy between the SWCNT and the hexane molecule for the DFT-D geometry optimised structure (where the optimisation began with the lowest energy structure obtained from the AMBER force field shown in Figure 1, lower panel) was $-26.5 \mathrm{~kJ}$ $\mathrm{mol}^{-1}$ using the $6-311 \mathrm{G}(\mathrm{d}, \mathrm{p})$ basis set, and -29.4 $\mathrm{kJ} \mathrm{mol}^{-1}$ using the $6-311 \mathrm{G}(2 \mathrm{~d}, 2 \mathrm{p})$ basis set. The AMBER binding energy for the structure shown in the lower panel of Figure 1 was $-34.1 \mathrm{~kJ} \mathrm{~mol}^{-1}$, which is slightly stronger compared to the DFT$D$ results. The distance between the centre of mass of the SWCNT and the hexane molecule according to the AMBER force field is $5.60 \AA$, which is slightly shorter than the DFT-D result of $5.89 \AA$ (both basis sets). The similarity between the results obtained from AMBER and DFT-D is a good indication that the AMBER force field provides reliable results for the SWCNT-alkane systems, such as those studied here. As described above, similar results are expected for systems consisting of graphene and hexane due to the similarity in composition and atom types of graphene and SWCNT systems.

\section{Density}

The density obtained after equilibration of the PE-graphene systems was $0.999 \pm 0.002 \mathrm{~kg} \mathrm{dm}^{-3}$ 
and $0.980 \pm 0.002 \mathrm{~kg} \mathrm{dm}^{-3}$ at $278 \mathrm{~K}$ and $328 \mathrm{~K}$, respectively. The corresponding values for the PE-SWCNT systems were $0.963 \pm 0.002 \mathrm{~kg} \mathrm{dm}^{-3}$ and $0.940 \pm 0.002 \mathrm{~kg} \mathrm{dm}^{-3}$. This is in good agreement with other studies ${ }^{35,50}$ and supports the validity of the AMBER force field. The lower density for the PE-SWCNT systems is due to the larger volume of the SWCNT compared to the graphene sheet. Densities were also calculated considering only the PE regions of each box. At $278 \mathrm{~K}$ the density of PE in the PE-graphene systems and the PE-SWCNT systems were 0.858 $\pm 0.005 \mathrm{~kg} \mathrm{dm}^{-3}$ and $0.854 \pm 0.005 \mathrm{~kg} \mathrm{dm}^{-3}$, respectively. This is slightly higher than the density of amorphous PE, which was calculated from MD simulations as $0.846 \pm 0.002 \mathrm{~kg} \mathrm{dm}$ $3,6,9$ but is similar to the experimental value of $0.855 \mathrm{~kg} \mathrm{dm}^{-3} .{ }^{50}$ Consequently, there is a closer packing of the polymer in the presence of the filler, which is in agreement with the simulation studies of Herasati et al., ${ }^{20}$ where the polymer had a more ordered structure. This more ordered structure obtained in the present simulations is shown in Figures 3 and 4.

As can be seen in Figures 3 and 4, addition of a graphene sheet or SWCNT induces a layered PE structure near the additive surface. The total density curves in Figure 3 have peaks that are separated by $\sim 4.5 \AA$. The height of these peaks decreases and the peaks broaden with increasing distance to the additive, which shows that the ordered structure decreases with increasing distance to the additive. At long distances (beyond the periodic box border of $\sim 15 \AA$ A) the layering diminishes and the PE structure is amorphous. At this stage the total density curve has a value of 1 .

Hence, purely amorphous PE is not obtained with the system sizes used here (indicated by the fact that the oscillations in the density profiles in Figure 3 do not diminish along the box length). However, purely amorphous PE systems have been studied in a previous study. ${ }^{6}$ Modelling of systems that include both the bulk and interphase structures of PE require far larger systems than those studied here and
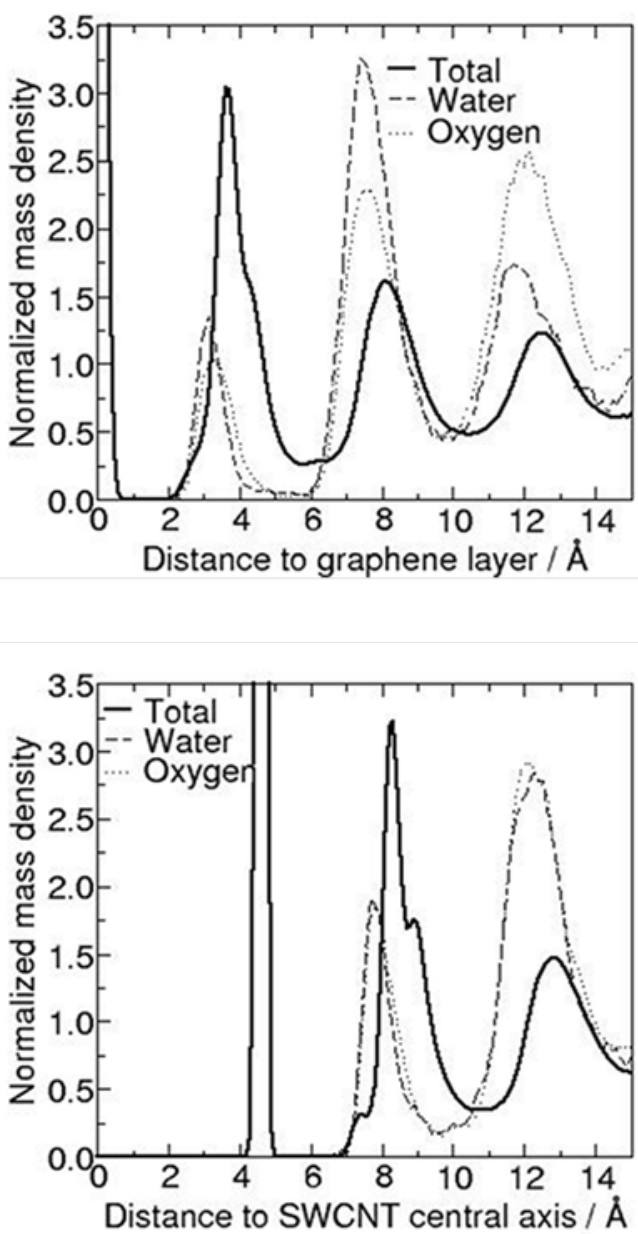

FIGURE 3. Density as a function of the distance to the graphene layer (upper panel) and the central axis of the SWCNT (lower panel). The local density of water (dashed) and oxygen (dotted) is normalized by the overall mass density of water and oxygen, respectively, in the periodic box. The density of all atoms (solid line) is normalized by the overall density for all atoms in the box. The density profiles are averaged from the simulations of the diffusion of water and oxygen at $298 \mathrm{~K}$.

consequently longer computation times. The systems studied here allow for the study of the isolated effect of graphene or SWCNT addition on the PE structure and on the solubility, diffusion and permeation coefficients of water and oxygen. The results obtained here will also 
be valid for larger box sizes (lower additive fractions) but the effect of the additive will be smaller.

The layered PE structure is obtained since atoms in the PE chains arrange themselves to be at the equilibrium distance from the highly ordered structure of the additive surface. According to Figure 3 , this distance is approximately $3.6 \AA$ which is in excellent agreement with the distance between the $(3,3)$ SWCNT and the hexane molecule in the lowest energy structure in Figure 1 (3.55 $\AA$ ). The agreement between the model used for validation of the AMBER force field and the PESWCNT composite system not only confirms that the model consisting of a $(3,3)$ SWCNT and a hexane molecule is sufficiently large for validation but also supports the assumption that the SWCNT chirality is of little importance for the type of study performed here. The first layer is the most structured one (highest and thinnest peak in Figure 3 ) due to the highly ordered structure of the additive.
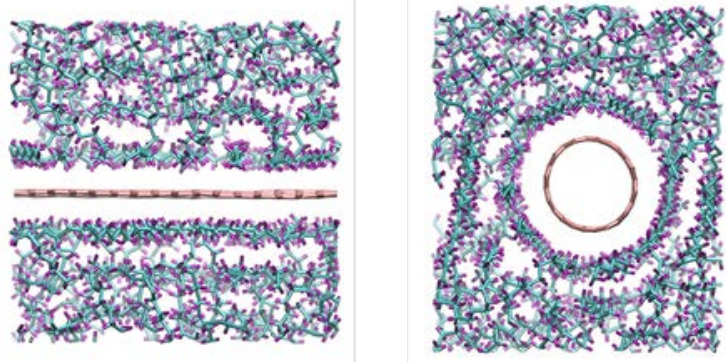

FIGURE 4. Layering in PE-graphene (left) and PESWCNT (right) systems.

Penetrant molecules are most likely to be found in cavities located in one of the layers of the PE or between the layers. As shown in Figure 3, the density of the penetrants is small in the polymer layers, which indicates that there are fewer (sufficiently large) cavities available in these regions (this is an effect of the ordered structure induced by the additives). Figure 5 shows typical snapshots from the MD simulations, which were taken when the penetrants were near the first PE layer (closest peak to the graphene/SWCNT surface in Figure 3). It is clear from these snapshots that the penetrants are located in PE cavities between the additive and the neighbouring PE layer. As seen in both Figures 3 and 5 , the penetrant molecules are located closer to the additives than the PE layers. This is due to their smaller diameter in comparison to $\mathrm{PE}$ and the strong attraction to the graphene or SWCNT surface. According to the Lorentz-Berthelot mixing rules, $^{55,56}$ and the AMBER force field, the L-J parameter $\sigma$ for the interaction of a graphene or SWCNT carbon atom and an oxygen atom (in the oxygen molecule) is $3.24 \AA$ and the equilibrium separation $\left(r_{0}\right)$ occurs at $3.64 \AA$, which agrees well with the first density peaks of the oxygen penetrants in Figure 3 (located at $\sim 3.3 \AA$ from the graphene or SWCNT surface). The second and third peaks of water and oxygen in Figure 3 are also dislocated slightly to the left compared to the PE peaks due to the presence of voids in this area and the interaction between the penetrant molecule and the more effectively packed PE layers close to the additive. For example, the value of the L-J equilibrium separation $\left(r_{0}\right)$ for a carbon backbone atom in one of the PE chains and an oxygen atom is $3.64 \AA$ which is in good agreement with the distance of $3.7 \AA$ between the first PE peak (e.g., located at $3.8 \AA$ for the graphene containing system) and the second density peak for the penetrants (located at 7.5 $\AA$ for the graphene containing system). 
a)

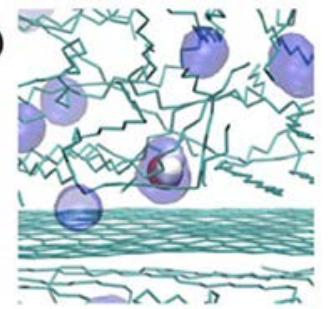

c)

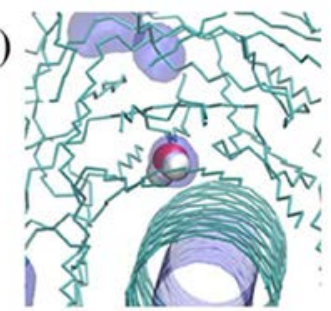

e)

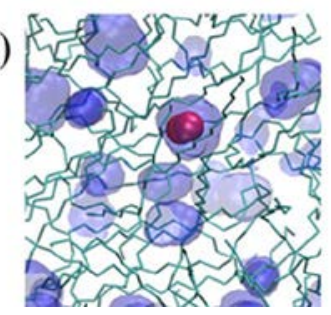

b)

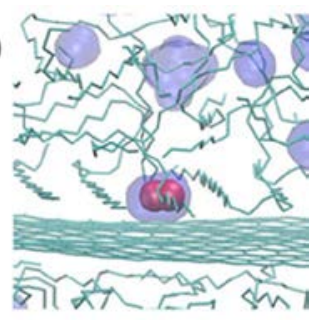

d)

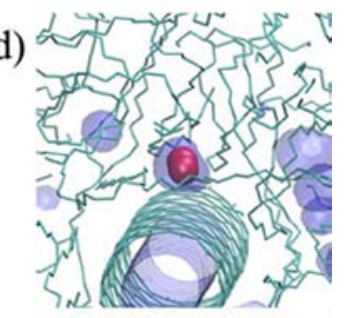

FIGURE 5. Snapshots from MD simulations of water $(a, c)$ and oxygen $(b, d)$ in PE-graphene $(a, b)$ and PE-SWCNT (c,d). Oxygen in amorphous $P E$ is shown in panel (e). In a-d the penetrant is near the first layer of $\mathrm{PE}$ relative to the additive. Colours (online): Carbon atoms in cyan, oxygen in red and hydrogen in white. The cavities obtained from a probe with radius $1.5 \AA$ are shown in blue.

The density profile for the SWCNT system given in Figure 3 is very similar to that of the graphene, given that the radius of the SWCNT is 4.6 $\AA$. This indicates that the effect on the PE structure is similar for both additives.

The angles between the normal of the graphene sheet and the bond vector of the penetrants were also calculated. At $278 \mathrm{~K}$, in $57 \%$ of 5740 snapshots analysed, was the angle between the $\mathrm{O}-\mathrm{O}$ bond in oxygen and the graphene normal between 60 and 90 degrees. Similarly, for water, $50 \%$ of the angles between either of the $\mathrm{H}-\mathrm{O}$ bonds and the normal to the sheet were

between 60 and 90 degrees. Hence, the penetrant molecules tend to lie parallel to the sheet, rather than perpendicular to it.

\section{Solubility}

Figure 6 shows the solubility coefficients (S) for water and oxygen in the graphene and SWCNT composites. The coefficients of water and oxygen in pure amorphous $\mathrm{PE}$, obtained from a previous study, ${ }^{6}$ are also shown. Since the additives occupy different volumes, the solubility coefficients are also given in units of $g$ penetrant per $\mathrm{g} P \mathrm{PE}$ in the inserts of Figure 6 . It is clear from Figure 6 that the change of units does not affect the ordering of solubility coefficients.

a)

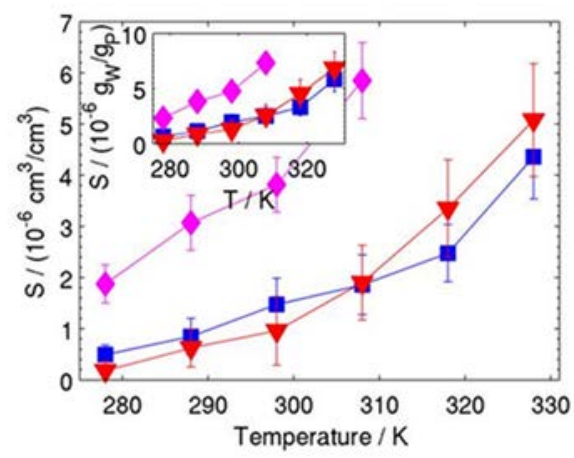

b)

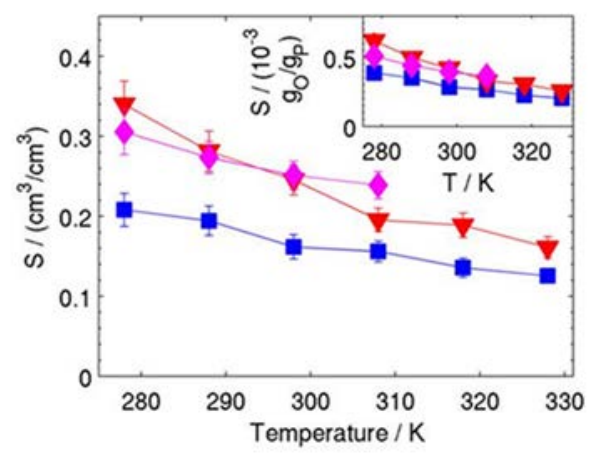

FIGURE 6. Solubility coefficients of water (a) and oxygen (b) in PE with no additive (magenta diamonds), graphene (red triangles) and SWCNT additive (blue squares). The error bars show the $95 \%$ confidence interval of the mean values. The inserts show the same data but in units of $g$ penetrant per $\mathrm{g} P \mathrm{PE}$. 
Figure 6a shows that the solubility coefficient of water is lower in the systems containing the additives, and that $\mathrm{S}$ is not significantly affected by the shape of the additive. Furthermore, the solubility coefficient of water increases with increasing temperature, which is in agreement with previous simulations ${ }^{6}$ and experimental studies. ${ }^{13}$

There is a significant difference between the effects of the two additives on the solubility coefficient of oxygen (Figure 6b). The presence of the graphene layer does not change the solubility coefficient significantly compared to amorphous PE. In fact, the presence of the graphene may even increase the solubility coefficient of oxygen at lower temperatures. In contrast, the presence of the SWCNT decreases $S$ compared to the amorphous $\mathrm{PE}$ in this temperature range.

Figure 6 reveals that it is only the combination of oxygen and graphene that results in solubility coefficients that are similar to the amorphous PE. For all other penetrant / additive combinations the solubility coefficient decreases relative to the amorphous PE. This can be explained by the phase of the penetrant and the binding strength between the penetrant and the additive. Water is in the liquid phase at the simulated pressuretemperature conditions whereas oxygen is in the gas phase (as discussed before, ${ }^{6}$ this is why the solubility coefficient of water increases with increasing temperature whereas the solubility coefficient of oxygen decreases). The hydrogen bonding between water molecules in the liquid phase is stronger than the bonding between the additive and water, and hence the additive will not attract water into the composite. The AMBER binding energy between a water molecule and a $(3,3)$ SWCNT (similar to the $(3$, 3)SWCNT used for the AMBER validation described above) is between -4.6 to $-5.0 \mathrm{~kJ} \mathrm{~mol}^{-}$ 1 . This is slightly less than previously reported interaction strengths of -6.8 to $-14.0 \mathrm{~kJ} \mathrm{~mol}^{-1}$ between a water molecule and graphene-like surfaces..$^{57,58}$ Consequently, the calculated solubility coefficient of water might be slightly underestimated and the diffusion coefficient slightly overestimated for the systems containing graphene or a SWCNT but the obtained trends in solubility and diffusion coefficients of water with increasing temperatures are not expected to be affected. In contrast, the intermolecular interaction between oxygen molecules is negligible in the gas phase (it behaves as an ideal gas), and the binding strength between the additive and the oxygen may therefore attract oxygen into the composite. In fact, this is seen for graphene at lower temperatures (where entropic effects do not dominate over the penetrant - graphene binding energies), but it is not seen for the SWCNT filler. The reason for this is the larger number of graphene $\mathrm{C}-$ oxygen $\mathrm{O}$ intermolecular interactions that can be obtained in these systems compared to the SWCNT systems. Since the graphene sheet is planar, each oxygen atom interacts with a larger number of carbon atoms in the graphene sheet compared to the SWCNTs. This is illustrated in Figure 7, where the number of atoms within the $14 \AA$ cutoff (used in the simulations) are shown in green. There are $174 \mathrm{C}$ green carbon atoms in the graphene sheet compared to 149 in the SWCNT.
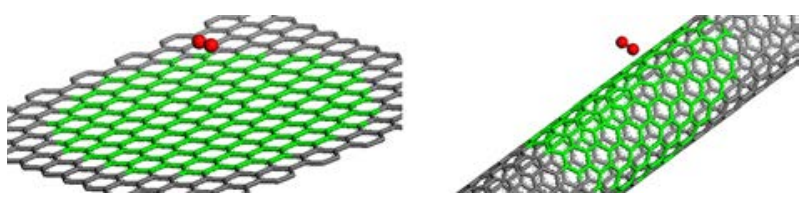

Figure 7. A comparison of the number of carbon atoms that can interact with the atoms of an oxygen molecule located $6.0 \AA$ above the surface of the additive. 174 atoms of the graphene sheet (shown in green in the left panel) interact with the oxygen atoms, whereas only 149 of the SWCNT atoms (right) interact with the oxygen atoms. The oxygen - carbon atom cutoff used to generate the figure is the same as that used in the simulations ( $14.0 \AA$ ). 
Hence, it is the large intermolecular interaction strength between the graphene sheet and the oxygen molecule that leads to the increased solubility coefficient due to the presence of graphene at low temperatures. Support for this hypothesis was obtained by repeating the oxygen solubility calculations at $278 \mathrm{~K}$ for the graphene systems, but with $\sigma_{\mathrm{c}-\mathrm{O}}=4.43 \AA$ and $\varepsilon_{\mathrm{C}-\mathrm{O}}=0.44 \mathrm{~K}$ (compared to the original values of $\sigma_{\mathrm{C}-\mathrm{O}}=3.24 \AA$ and $\varepsilon_{\mathrm{C}-\mathrm{O}}=43.93 \mathrm{~K}$ ). These $\sigma$ and $\varepsilon$ were chosen so that the potential well was 100 times shallower than the value used in the simulations at the same time that $V(r)$ has a similar repulsion at distances relevant to the simulation conditions.

The smaller interaction strength resulted in a solubility coefficient of $0.23 \mathrm{~cm}^{3} \mathrm{~cm}^{-3}$, compared to the original value of $0.34 \mathrm{~cm}^{3} \mathrm{~cm}^{-3}$. In fact, the lower solubility coefficient is similar to that obtained in the presence of the SWCNT $(0.21$ $\mathrm{cm}^{3} \mathrm{~cm}^{-3}$ ). As mentioned above, the effect of the large graphene - oxygen interaction strength are most significant at lower temperatures where the enthalpic properties dominate over the entropic contributions (at higher temperatures the oxygen molecules desorb from the composite and enter into the gas phase oxygen box).

It should be reiterated that the systems do not contain any crystal regions. The graphene or SWCNT induces an increased ordered structure in the surrounding PE. This ordered structure influences the $S$ and $D$ (and consequently $P$ ) but cannot be regarded as a crystalline structure. PE crystallites have an orthorhombic unit cell and the arrangement of the chains ${ }^{59,60}$ do not resemble the ordering obtained as a function of the graphene or SWCNT addition. Furthermore, it is often assumed that the crystalline regions of semicrystalline polymers are inaccessible to gas and liquid penetrants due to the close packing of the polymer chains. As shown in Figure 5 there exist cavities that can act as adsorption sites for oxygen and water even in this ordered structure of PE.

Plots of the dependence of $\ln (S)$ on the inverse of the temperature that were used to calculate the heat of solution $\left(\Delta \mathrm{H}_{\mathrm{s}}\right)$, entropy of solution $\left(\Delta \mathrm{S}_{\mathrm{s}}\right)$ and the Gibbs energy of solution $\left(\Delta \mathrm{G}_{\mathrm{s}}\right)$ are shown in Figure 8 and the calculated values are listed Table 1 . The absorption of water in PE is an endothermic process and the heat of solution of water increases in the presence of an additive in the following order: $\mathrm{PE}<\mathrm{PE}-$ SWCNT < PE-graphene. That is, the solution process is more endothermic for water when there are additives present. The endothermicity of water adsorption is probably due to the fact that strong hydrogen-bonds between water molecules need to be broken when water moves from the liquid phase into the polymer. The increase in endothermicity due to the additives may be directly due to their presence or to the restructuring of the PE that they induce.

The absorption of oxygen in PE is exothermic, and the heat of solution becomes more exothermic in the presence of the additives. In contrast to water, oxygen is in the gas phase and formation of oxygen-PE and oxygen-filler intermolecular bonds will release energy. As discussed above, there is a larger interaction strength between graphene and oxygen then between SWCNT and oxygen, and this increases the exothermicity of the dissolution.

As can be seen in Table 1 below, the error bars for $\Delta S_{s}$ and $\Delta G_{s}$ are too large to allow for statistically meaningful conclusions. 
a)

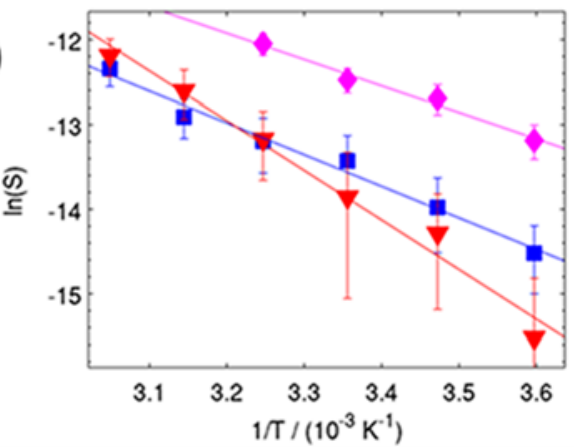

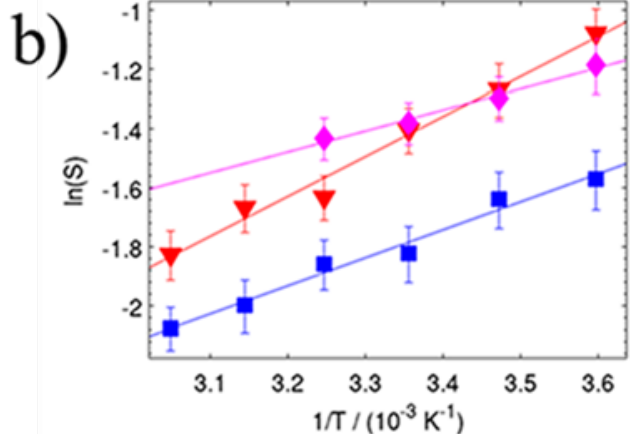

FIGURE 8. Solubility coefficient temperature dependence of water (a) and oxygen (b) for PE with no additive (magenta diamonds), graphene (red triangles) and SWCNT additive (blue squares). The error bars show the $95 \%$ confidence interval of the mean values.

TABLE 1. Heats of solution, entropy of solution and Gibbs free energy of solution at $298 \mathrm{~K}$ for water and oxygen dissolution in the PE and the PE nanocomposites.

\begin{tabular}{l|l|l|l|l|l|l}
\hline & \multicolumn{5}{|l|}{$\mathrm{H}_{2} \mathrm{O}$} & \multicolumn{2}{l}{$\mathrm{O}_{2}$} \\
& $\begin{array}{l}\Delta \mathrm{H}_{\mathrm{s}} \\
(\mathrm{kJ} / \mathrm{mol})\end{array}$ & $\begin{array}{l}\Delta \mathrm{S}_{\mathrm{s}} \\
(\mathrm{J} / \mathrm{K} \text { mol })\end{array}$ & $\begin{array}{l}\Delta \mathrm{G}_{\mathrm{s}} \\
(\mathrm{kJ} / \mathrm{mol})\end{array}$ & $\begin{array}{l}\Delta \mathrm{H}_{\mathrm{s}} \\
(\mathrm{kJ} / \mathrm{mol})\end{array}$ & $\begin{array}{l}\Delta \mathrm{S}_{\mathrm{s}} \\
(\mathrm{J} / \mathrm{K} \mathrm{mol})\end{array}$ & $\begin{array}{l}\Delta \mathrm{G}_{\mathrm{s}} \\
(\mathrm{kJ} / \mathrm{mol})\end{array}$ \\
\hline Graphene & $49 \pm 10$ & $48 \pm 32$ & $34 \pm 20$ & $-11 \pm 3$ & $-49 \pm 8$ & $4 \pm 5$ \\
\hline SWCNT & $31 \pm 6$ & $-8 \pm 19$ & $34 \pm 12$ & $-8 \pm 2$ & $-41 \pm 6$ & $4 \pm 4$ \\
\hline No filler & $26 \pm 11$ & $-16 \pm 37$ & $31 \pm 22$ & $-6 \pm 3$ & $-31 \pm 9$ & $3 \pm 6$ \\
\hline
\end{tabular}

\section{Diffusion}

The diffusion of water and oxygen through PE is hindered by the presence of the graphene layer or the SWCNT. The MSD for water at $328 \mathrm{~K}$ in the $x, y$ and $z$ directions as well as the total MSD in both composite systems are displayed in Figure 9. Similar diagrams are obtained for oxygen diffusion and at the other temperatures. The graphene layer is located in the $x-y$ plane of the periodic box and, due to the periodic nature of the graphene, diffusion perpendicular to the layer (i.e. in the z-direction) is hindered completely at longer simulation times. This is seen in the left diagram of Figure 9. Future simulations of larger systems with finite size graphene sheets will provide further insights into the diffusion in the direction perpendicular to the graphene plane.

The SWCNT is aligned along the z-axis of the periodic box, and hence the diffusion in the $x$ and $y$ directions is hindered due to the tortuous path that the penetrants need to take around the SWCNT. As expected, and as can be seen in the right panel of Figure 9, the MSDs in the $x$ and $y$ directions are smaller compared to the MSD in the z-direction for the PE-SWCNT composite. The total MSD is very similar for both composites.

The calculations of the diffusion coefficients were performed with one penetrant molecule inserted into the composite systems as described above. According to the results 
obtained from the calculations of the solubility coefficients the numbers of water and oxygen molecules in the composite systems are on average less than one at an external pressure of 101.325 kPa. However, when calculating the diffusion coefficients at least one penetrant molecule must be present. At an external pressure of $500 \mathrm{kPa}$ there is on average one oxygen molecule present in the composite systems. For water, external pressures of 10 GPa still results in less than one molecule on average in the composite systems.

The temperature dependence of the diffusion coefficients parallel to the additives, i.e., in the $x-y$ plane for the PE-graphene systems and along the $z$ axis for the PE-SWCNT systems is displayed in Figure 10. The diffusion in amorphous PE obtained in a previous study is also shown for comparison. ${ }^{6}$ The diffusion of water and oxygen in these directions does not change significantly in the presence of the additive. D was calculated for 144 individual systems at each temperature (with one water molecule inserted into each system). The number of systems together with the small error bars, as well as the monotonic trends of increasing $D$ with increasing temperature (Figure 10), indicate that the calculations are converged.

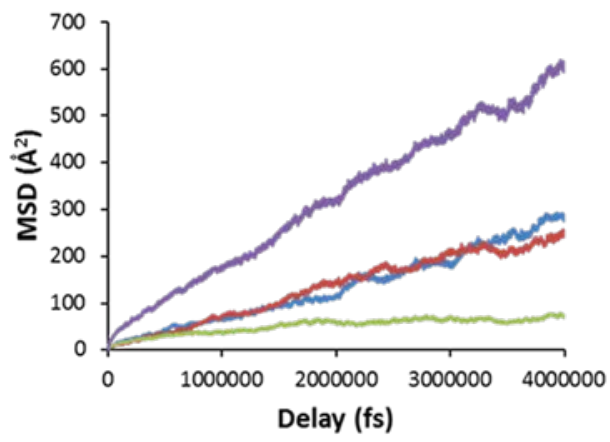

Figure 11 shows the local diffusion coefficients (at various distances from the graphene sheet) for different temperatures. The distance to the graphene layer was divided into three intervals which are related to the density peaks of the penetrant molecules in Figure 3 (2.0-5.5 $\AA$, 5.5$10.5 \AA$ and $10.5-15.0 \AA$ ). Diffusion increases with increasing distance from the additive for both water and oxygen. This is because the structure of PE is more disordered and mobile at larger distances from the additive and also due to the decreased interaction between the penetrants and the graphene or SWCNT. The diffusion coefficients calculated with this method is generally larger than the coefficients presented above. As discussed above, this is due to the shorter time intervals (500 ps) used when calculating the MSD.

\section{Permeation}

The difference in permeation coefficients of water and oxygen in the two composite systems follows the same trend as the solubility coefficients since the diffusion coefficients in the systems are similar. Hence, as can be seen in Figure 12, the permeation coefficient of water decreases when either of the additives

FIGURE 9. Mean square displacement (MSD) of water in different directions in the PE-graphene (left) and the PE-SWCNT (right) composite at $328 \mathrm{~K}$.

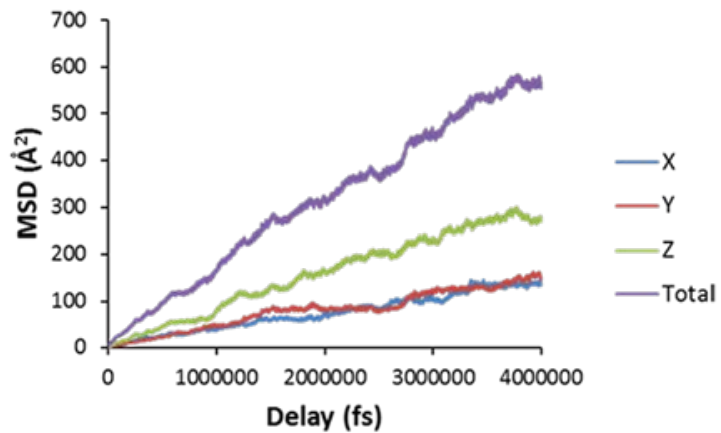


a)

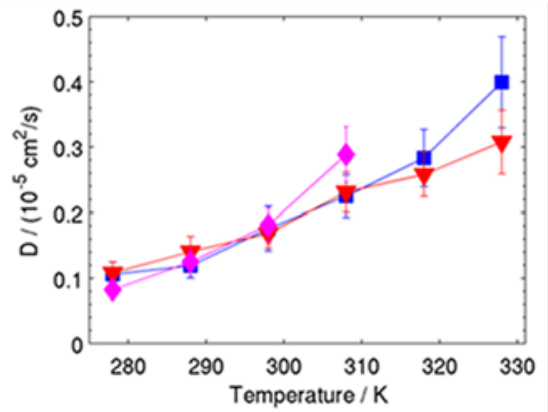

b)

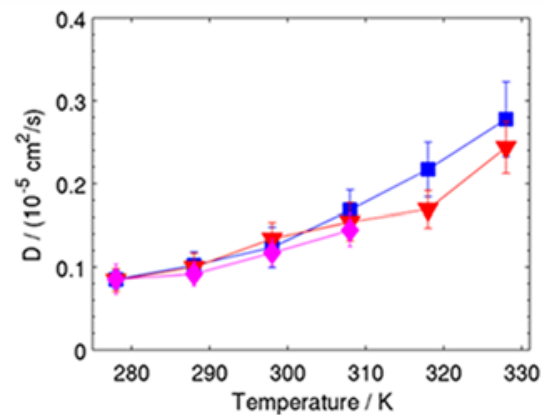

FIGURE 10. Diffusion of water (a) and oxygen (b) parallel to the graphene (red triangles), SWCNT (blue squares) and in pure PE (magenta diamonds). The error bars show the $95 \%$ confidence interval of the mean values.

a)

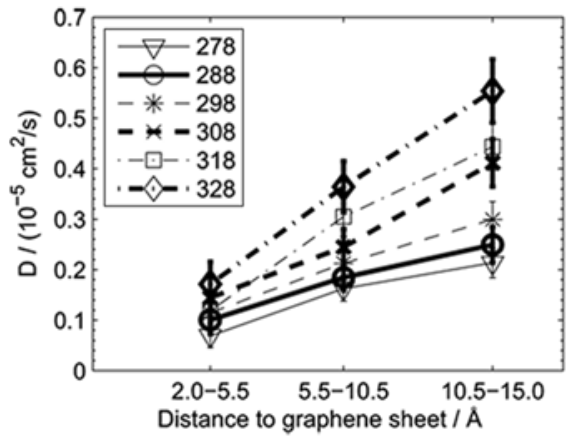

b)

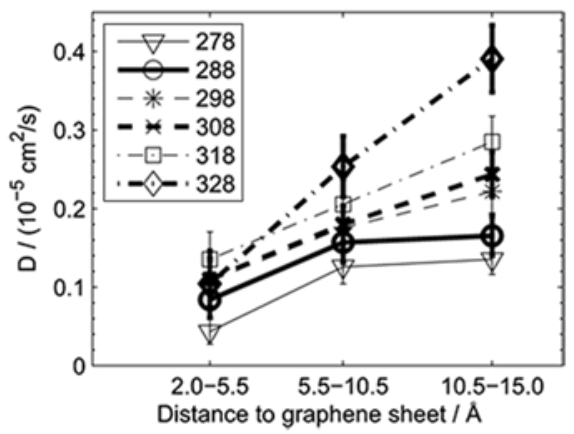

FIGURE 11. Local diffusion coefficients of water (a) and oxygen (b) parallel to the graphene layer at different temperatures. The diffusion is shown at different distances to the graphene layer, corresponding to the peaks in Figure 3. The error bars show the $95 \%$ confidence interval of the mean values.

are introduced to the system, while the permeation coefficient of oxygen decreases when adding the SWCNT but not when adding

a)

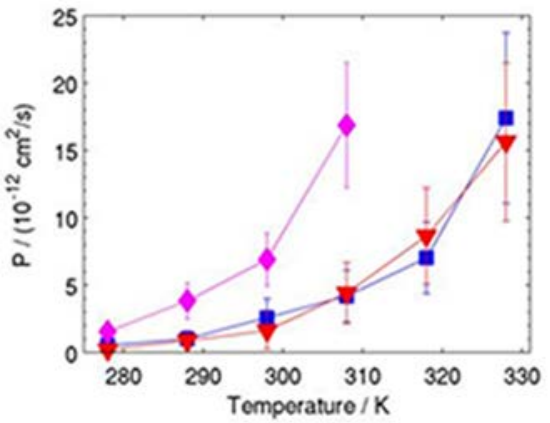

the graphene sheet. In fact, addition of the graphene sheet may increase permeation at

b)

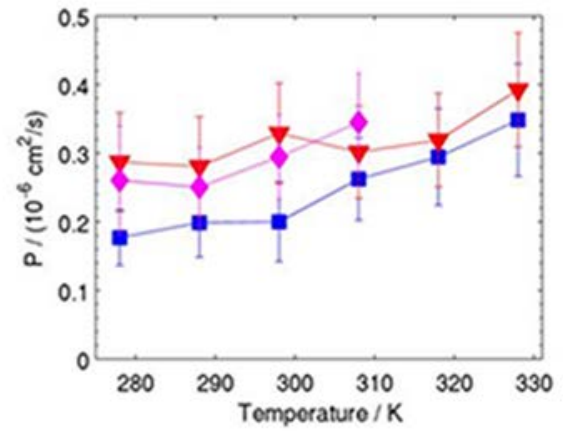

FIGURE 12. Permeation coefficients of water (a) and oxygen (b) as a function of temperature for PE with no additive (magenta diamonds), graphene (red triangles) and SWCNT additive (blue squares). The error bars show the $95 \%$ confidence interval of the mean values. 
lower temperatures. This indicates that addition of graphene nanofillers can selectively increase the permeation coefficient of oxygen compared to water.

\section{CONCLUSIONS}

$M C$ and MD simulations have been performed on composites consisting of PE and graphene or PE and SWCNTs to calculate the solubility, diffusion and permeation coefficients of water and oxygen in these systems.

Only amorphous systems have been considered since the introduction of crystallites requires far larger model systems and computation times. Also, due to the close packing of the polymer chains in the crystallites the solubility and diffusion in these regions are negligible in comparison to the amorphous regions. Inclusion of graphene or SWCNTs results in an increased ordering of the PE chains in the vicinity of the additives.

The solubility coefficient of water decreases with the addition of either graphene or SWCNTs while the effect on the solubility coefficient of oxygen is dependent on the type of additive. The solubility coefficient of oxygen decreases with the addition of SWCNTs but graphene addition does not alter the solubility coefficient significantly compared to amorphous PE. In fact, the presence of graphene may even increase the solubility coefficient at lower temperatures. The effect is related to the phase of the penetrant molecule (liquid or gas) and the intermolecular interaction strength between the penetrant and the additive.

The diffusion coefficients of water and oxygen do not change significantly in the direction parallel to the additive compared to pure amorphous PE. However, smaller diffusion coefficients in this direction are obtained when the penetrant molecules are located close to the surface of the additives. This is due to the closer packing of the PE molecules in this region.
Combining the solubility and diffusion results yields a permeation coefficient for water that decreases when graphene or SWCNTs fillers are added to the PE. The effect is more distinct as the temperature increases from 278 to $308 \mathrm{~K}$. In contrast, the permeability of oxygen decreases when SWCNTs are used as fillers, but not when graphene is used. Hence graphene can be added to PE to increase the permeation rate of oxygen relative to water.

\section{ACKNOWLEDGEMENTS}

The authors gratefully acknowledge the Swedish Knowledge Foundation (KK-stiftelsen) for financial support. The simulations were performed on resources provided by the Swedish National Infrastructure for Computing (SNIC) at the High Performance Computing Centre North (HPC2N) and the National Supercomputer Centre (NSC) in Linköping.

\section{REFERENCES}

1. V. Neburchilov, J. Martin, H. Wang, J. Zhang, J. Power Sources, 2007, 169, 221-238.

2. P. Bernardo, E. Drioli, G. Golemme, Ind. Eng. Chem. Res. 2009, 48, 4638-4663.

3. J. Gajdoš, K. Galić, Ž. Kurtanjek, N. Ciković, Polym. Test. 2000, 20, 49-57.

4. A. J. Peacock, In Handbook of Polyethylene - Structures, Properties, and Applications; Marcel Dekker Inc., New York, Basel, 2000.

5. R. J. Ashley, In Permeability and Plastics Packaging in Polymer Permeability; J. Comyn, Ed.; Chapman \& Hall, London, 1985, pp 269-308.

6. A. Börjesson, E. Erdtman, P. Ahlström, M. Berlin, T. Andersson, K. Bolton, Polymer 2013, 54, 2988-2998.

7. A. Al-Jabareen, H. Al-Bustami, H. Harel, G. Marom, J. Appl. Polym. Sci. 2013, 128, 1534-1539.

8. Y. Li, Polymer 2011, 52, 2310-2318. 
9. A. Adnan, C. T. Sun, H. Mahfuz, Compos. Sci. Technol. 2007, 67, 348-356.

10. A. S. Michaels, H. J. Bixler, J. Polym. Sci. 1961, 50, 393-412.

11. A. S. Michaels, H. J. Bixler, J. Polym. Sci. 1961, 50, 413-439.

12. V. Compañ, A. Ribes, R. Díaz-Calleja, E. Riande, Polymer 1996, 37, 2243-2250.

13. D. W. McCall, D. C. Douglass, L. L. Blyler, G. E. Johnson, L. W. Jelinski, H. E. Bair, Macromolecules 1984, 17, 1644-1649.

14. S. Mrkić, K. Galić, M. Ivanković, S. Hamin, N. Ciković, J. Appl. Polym. Sci. 2006, 99, 1590-1599.

15. M. Kurek, D. Klepac, M. Ščetar, K. Galić, S. Valić, Y. Liu, W. Yang, Polym. Bull. 2011, 67, 1293-1309.

16. P. Gestoso, N. Ch. Karayiannis, J. Phys. Chem. B 2008, 112, 5646-5660.

17. P. Schatzberg, J. Phys. Chem. 1963, 67, 776-779.

18. A. Arora, G. W. Padua, J. Food Sci. 2010, 75, R43-R49.

19. B. M. Yoo, H. J. Shin, H. W. Yoon, H. B. Park, J. Appl. Polym. Sci. 2014, 131, 39628.

20. S. Herasati, H. H. Ruan, L. C. Zhang, J. Nano Res. 2013, 23, 16-23.

21. C. Wei, D. Srivastava, K. Cho, Los Alamos National Laboratory, Preprint Archive, Condensed Matter, 2002, 1-11.

22. A. Karatrantos, R. J. Composto, K. I. Winey, N. Clarke, Macromolecules 2011, 44, 9830-9838.

23. L. Sijia, Z. Wanxi, Y. Weiguo, S. Tongfei, Chem. Res. Chin. Univ. 2015, 31, $477-$ 483.

24. C. Batistakis, A. V. Lyulin, M. A. J. Michels, Macromolecules 2012, 45, 7282-7292.

25. M. Erber, A. Khalyavina, K.-J. Eichhorn, B. I. Voit, Polymer 2010, 51, 129-135.

26. E. T. Thostenson, Z. Ren, T.-W. Chou, Compos. Sci. Technol. 2001, 61, 18991912.

27. T. Gkourmpis, In Nanoscience and computational chemistry: Research progress; G. A. Mercader, E. A. Castro,
A. K. Haghi, Eds.; Apple Academic Press, Inc., 2014.

28. P. Pötschke, M. Abdel-Goad, I. Alig, S. Dudkin, D. Lellinger, Polymer 2004, 45 8863-8870.

29. T. Gkourmpis, C. Svanberg, S. K. Kaliappan, W. Schaffer, M. Obadal, D. Tranchida, Eur. Polym. J. 2013, 49, 1975-1983.

30. K. Chrissafis, K. M. Paraskevopoulos, I. Tsiaoussis, D. Bikiaris, J. Appl. Polym. Sci. 2009, 114, 1606-1618.

31. P. A. Song, Y. Yu, T. Zhang, S. Fu, Z. Fang, Q. Wu, Ind. Eng. Chem. Res. 2012, 51, 7255-7263.

32. Ö. Yargı, Ş. Ugur, Ö. Pekcan, Polym. Eng. Sci. 2012, 52, 172-179.

33. W. D. Cornell, P. Cieplak, C. I. Bayly, I. R. Gould, K. M. Merz, D. M. Ferguson, D. C. Spellmeyer, T. Fox, J. W. Caldwell, P. A. Kollman, J. Am. Chem. Soc. 1995, 117, 5179-5197.

34. LAMMPS 12 Mar 2011, Sandia National Labs, Albuquerque, NM, USA, http://lammps.sandia.gov, 2011.

35. S. Haghighatpanah, K. Bolton, Comp. Mater. Sci. 2013, 69, 443-454.

36. H. Sun, J. Phys. Chem. B 1998, 102, 7338-7364.

37. A. D. Becke, J. Chem. Phys. 1993, 98, 5648-5652.

38. S. Grimme, J. Antony, S. Ehrlich, H. Krieg, J. Chem. Phys. 2010, 132, 154104.

39. R. Peverati, K. K. Baldridge, J. Chem. Theory Comput. 2008, 4, 2030-2048.

40. J. Moellmann, S. Grimme, Phys. Chem. Chem. Phys. 2010, 12, 8500-8504.

41. M. Bohlén, K. C. Satyanarayana, K. Bolton, J. Comput. Theor. Nanos. 2013, 10, 1317-1325.

42. M. Bohlén, K. Bolton, Quantum Matter 2014, 3, 339-343.

43. M. W. Schmidt, K. K. Baldridge, J. A. Boatz, S. T. Elbert, M. S. Gordon, J. H. Jensen, S. Koseki, N. Matsunaga, K. A. Nguyen, S. Su, T. L. Windus, M. Dupuis, J. A. Montgomery, J. Comput. Chem. 1993, 14, 1347-1363. 
44. GAMESS (US), 20101001R1, lowa State University Quantum Chemistry Group, Ames,

IA, USA, http://www.msg.chem.iastate.edu/gam ess/index.html, 2007.

45. M. G. Martin, MCCCS Towhee, 6.2.15; http://towhee.sourceforge.net, 2011.

46. E. Johansson, K. Bolton, D. N. Theodorou, P. Ahlstrom, J. Chem. Phys. 2007, 126, 224902.

47. A. Jorio, R. Saito, J. H. Hafner, C. M. Lieber, M. Hunter, T. McClure, G. Dresselhaus, M. S. Dresselhaus, Phys. Rev. Lett. 2001, 86, 1118-1121.

48. D. Chen, M. Wang, W.-D. Zhang, T. Liu, J. Appl. Polym. Sci. 2009, 113, 644-650.

49. K. Ke, R. Wen, Y. Wang, W. Yang, B.-H. Xie, M.-B. Yang, J. Mater. Sci. 2011, 46, 1542-1550.

50. G. Allen, G. Gee, G. J. Wilson, Polymer 1960, 1, 456-466.

51. S. Nosé, J. Chem. Phys. 1984, 81, $511-$ 519.

52. W. G. Hoover, Phys. Rev. A 1985, 31, 1695-1697.

53. K. Bolton, S. Nordholm, J. Comput. Phys. 1994, 113, 320-335.

54. N. Tarantino, J.-Y. Tinevez, E. F. Crowell, B. Boisson, R. Henriques, M. Mhlanga, F. Agou, A. Israël, E. Laplantine, J. Cell Biol. 2014, 204, 231-245.

55. H. A. Lorentz, Annalen der Physik 1881, 248, 127-136.
56. D. Berthelot, Comptes rendus hebdomadaires des séances de l'Académie des Sciences 1889, 17031708.

57. J. Ma, A. Michaelides, D. Alfè, L. Schimka, G. Kresse, E. Wang, Phys. Rev. B 2011, 84, 033402.

58. G. R. Jenness, O. Karalti, W. A. Al-Saidi, K. D. Jordan, J. Phys. Chem. A 2011, 115, 5955-5964.

59. G. D. Barrera, S. F. Parker, A. J. RamirezCuesta, P. C. H. Mitchell, Macromolecules 2006, 39, 2683-2690.

60. A. Calzolari, T. Jayasekera, K. W. Kim, M. B. Nardelli, J. Phys.: Condens. Matter 2012, 24, 492204 


\section{GRAPHICAL ABSTRACT}

Edvin Erdtman, Martin Bohlén, Peter Ahlström, Thomas Gkourmpis, Mikael Berlin, Thorbjörn Andersson, Kim Bolton

\section{A MOLECULAR-LEVEL COMPUTATIONAL STUDY OF THE DIFFUSION AND SOLUBILITY OF WATER AND OXYGEN IN CARBONACEOUS POLYETHYLENE NANOCOMPOSITES}

Studying water and oxygen permeation through polyethylene-based materials is important for many applications, food packaging being one. Results from molecular simulations show that the permeation of these substances can be altered with the addition of either graphene or carbon nanotubes to polyethylene. A nanocomposite material that allows for the increased permeation rate of oxygen relative to water can be obtained.

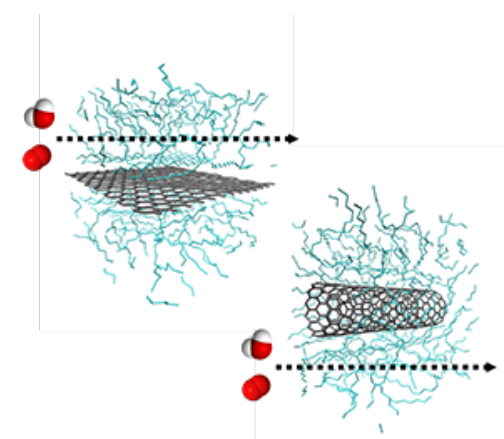

